\title{
BILATERAL MAXILLARY AND MANDIBULAR NERVE BLOCKS FOR POST-OPERATIVE PAIN MANAGEMENT IN MAXILLOFACIAL SURGERY FOR TREACHER-COLLINS SYNDROME: A CASE- REPORT
}

Lopes, Luís; Portugalyan, Mihran; Rodrigues, Idalina.

Hospital de Santa Maria, Departamento de Anestesiologia. Head: Prof. Dr. Lucindo Ormonde

Introduction. We present the case of a 17 year-old female patient with Treacher-Collins Syndrome, proposed for extensive maxillary osteotomy as well as mandibular orthognathic surgery. We detail the anaesthetic management, with particular focus on a multimodal and unconventional approach to pain management including multiple facial blocks, which we propose could represent a safe, effective and unburdensome protocol for dealing with maxillofacial surgery in a variety of patient profiles.

\section{The patient.}

> A 17 year-old female with no relevant medical history and a previous surgical facial intervention without complications.

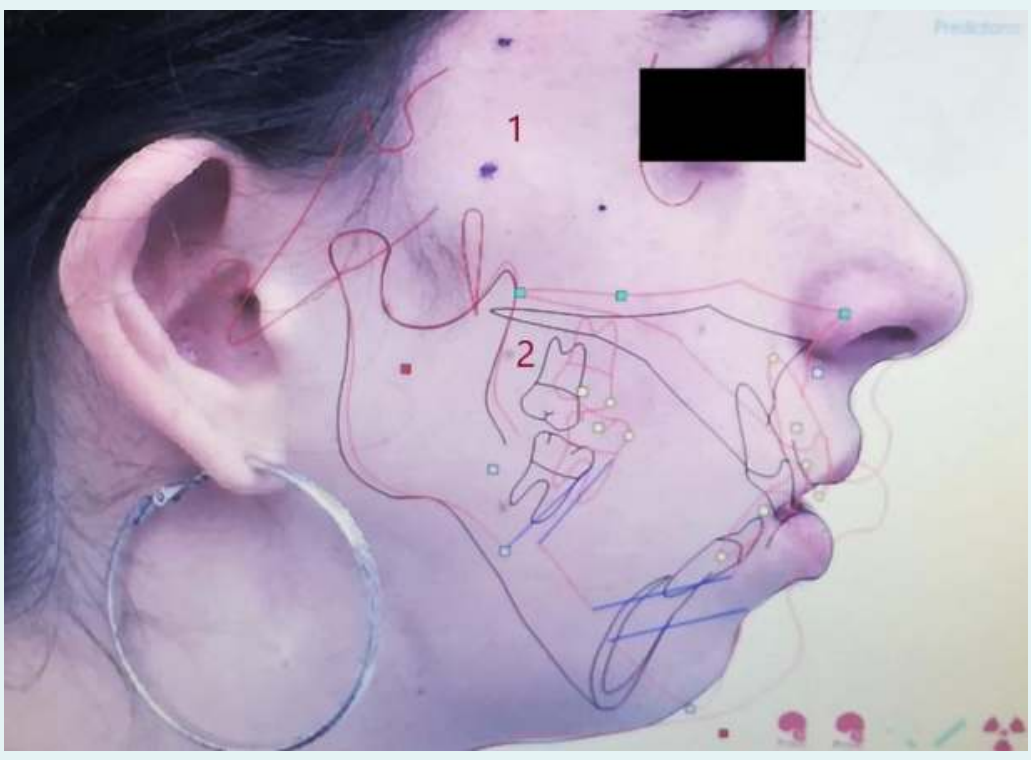

Figure 1. Lateral view of the pre-surgical anatomic details of the patient, with projected rendering of bone structures. Represented are the approximate puncture sites for the application of maxillary (1) and mandibular (2) blocks.

\section{Nerve block methodology}

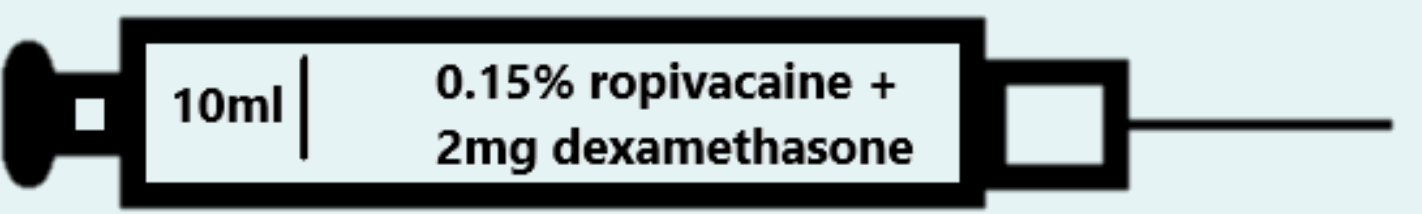

$2 \mathrm{ml}$ at each site

\section{Maxillary.}

> Suprazygomatic landmark approach: angle formed by the superior edge of the zygomatic arch below and the posterior orbital rim forward; insertion of $25 \mathrm{G}$ IM needle perpendicular to the skin and advanced to reach the greater wing of the sphenoid; caudal and posterior reorientation and further $(3 \mathrm{~cm})$ introduction to reach the pterygopalatine fossa.

\section{Mandibular.}

$>$ Ultrasound guidance combined with landmark knowledge and nerve stimulation

$>$ Ecography particularly useful due to the variant anatomy.

$>$ Probe placement ${ }^{2,3}$ : oblique position below the zygoma and transverse and slight anteriorly to the mandible and masseter muscle; identification of the lateral pterygoid plate and ideally the maxillary artery and nerve. In-plane insertion of $22 \mathrm{G}$ needle, in an anterior-to-posterior direction (point 2, figure 1).

\section{Treacher-Collins Syndrome.}

$>$ Autosomal dominant condition with variable expressivity, caused by variable mutations in the TCS gene (chromosome 5q31.3-q33.3),

$>$ Musculoskeletal abnormalities in the regions of the first and second branchial arches ${ }^{1}$ :

$>$ Hypoplasia of the malar bone, the maxilla and mandible, anterior open bite, retrognatia (as seen in this case, figure 1 ), etc.

The plan.

$>$ Total intravenous anaesthesia with propofol and remifentanyl TIVA.

$>$ Analgesia by means of bilateral maxillary and mandibular blocks. IV paracetamol + magnesium metamizole

$>$ Airway management by use of videolaringoscope-assisted intubation, fibroscopic backdrop availability

\section{Unrelated complication- an unexpected additional anaesthetic challenge \\ $>$ Surgical field haemorrhage with alveolar leakage and unilateral atelectasis despite throat packing and absence of cuff-leak: presented as delayed awakening and respiratory distress with asymmetrical thoracic excursion. \\ > Solved by fibroscopic aspiration and alveolar lavage with immediate full recovery}

\section{Outcomes at $48 \mathrm{~h}$ follow-up.}

$>$ Minimal reported pain $(\mathrm{VAS}<3$ )

$>$ No rescue opioid requirements.

$>$ Facial numbness reported as tolerable

$>$ Rapidly subsiding face swelling (figures 2 and 3).
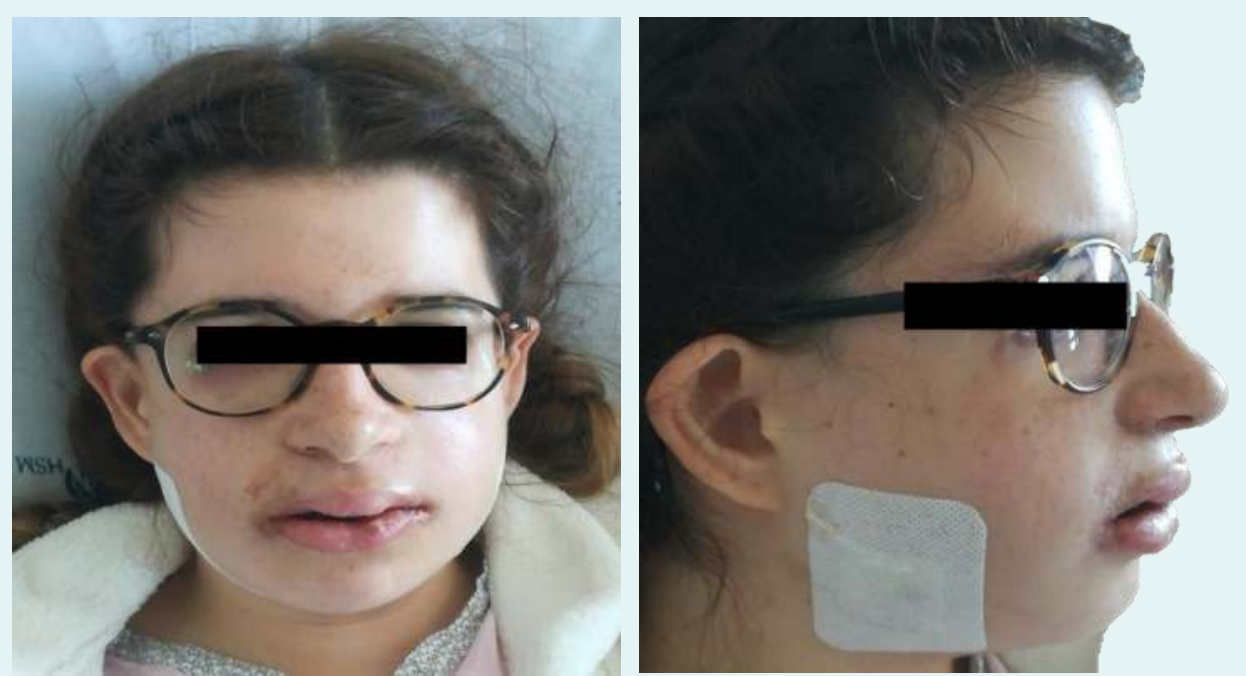

Figures 2 and 3. Post-operative day 1 lateral and frontal pictures of the patient.

Conclusions.

$>$ Extensive facial surgery might prove challenging in terms of anaesthetic management. Such challenges include haemodynamic control, airway securement and pain management.

$>$ In that regard, nerve blocks should be used with caution, and ideally making use of guidance methods such as ultrasound or nerve-stimulators, given the potentially altered anatomy.

$>$ We propose that a combined general plus regional anaesthetic technique, using low concentrations of local anaesthetic, might provide adequate analgesic coverage whilst minimizing side-effects.

> Further comparative studies are needed to access optimal dosage and possible effects of dexamethasone not only in extending block duration ${ }^{4}$, but also in minimizing tissue edema ${ }^{5}$ during the immediate post-operative period. 